\title{
Efecto de la proporción de genes Bos indicus $x$ Bos taurus sobre peso al destete y edad a primer parto en una población multirracial
}

\author{
Bos indicus $\mathbf{x}$ Bos taurus gene proportion over \\ weaning weight and age at first parturition in a \\ multi-breed population
}

Hugo O. Toledo Alvaradoa, J osé M. Berruecos Villalobosa, Carlos G. Vázquez Peláeza

\begin{abstract}
RESUMEN
Se analizaron 1,289 registros de hembras de primer parto con diversas proporciones de genes Bos indicus y Bos taurus (Charolais, Suizo, Simmental, Holstein Friesian y Salers). Tanto animales puros y cruzados de un hato comercial, ubicado en el municipio de Hueytamalco, Puebla, nacidas entre 1966 a 2006, con el objetivo de estimar la combinación óptima de genes Cebú y la retención de heterosis (RVH) sobre las características de peso al destete ajustado a 270 días (PD) y edad a primer parto (EPP). A partir de modelos de regresión múltiple se identificó la proporción de Cebú con el mejor comportamiento para las dos características de acuerdo al coeficiente de determinación $\left(R^{2}\right)$ y al estadístico de Mallow (CP). La mejor respuesta para PD se encontró en el rango de $\mathbf{4 2}$ a $70 \%$ de genes Bos indicus; mientras que las menores EPP se establecieron entre $\mathbf{2 7}$ al $\mathbf{4 0} \%$ de proporción Cebú. La retención de heterosis que mostró mayor potencial para PD fue de 76 a 78 y para EPP de 79 a $92 \%$. Estos resultados manifiestan la importancia de los efectos no aditivos en ambas características, así como la necesidad de realizar cruzamientos dirigidos.
\end{abstract}

PALABRAS ClAVE: Heterosis, Retención de heterosis, Cebú, Peso al destete, Edad a primer parto, Bovino.

\begin{abstract}
Records of 1,289 cows at first calving with different proportions of Bos Indicus and Bos Taurus genes (Charolais, Brown Swiss, Simmental, Holstein Friesian, Salers) were analyzed. The cows were both purebreds and crossbreds from a commercial herd born between 1966 and 2006. They were used to estimate the Zebu genes optimum combination on weaning weight adjusted to $\mathbf{2 7 0} \mathrm{d}$ (WW) and age at first calving (AFC) in the tropics of México. Likewise, heterosis retention (RVH) and their contribution to WW and AFC, was estimated. Multiple regressions analysis was used to select the best two, three or more crossbreed's model for each trait, according to the square of the multiple correlation coefficients $\left(R^{2}\right)$ and the Mallow statistic (CP). The results showed higher heterosis for WW between 42 to $70 \%$ of Bos indicus genes. On the other hand, the best response to heterosis for a lower AFC was found in a proportion of Zebu genes ranging from 27 to $\mathbf{4 0} \%$. The retention of heterosis showed that the greatest potential for WW was 76 to $78 \%$ and 79 to $92 \%$ for AFC. These results demonstrate the importance of non-additive effects in both traits, and the need for controlled crosses.
\end{abstract}

KEY WORDS: Heterosis, Retention of heterosis, Zebu, Weaning weight, Age at first calving, Bovine.

\section{INTRODUCCIÓN}

Es común observar que los productores en las regiones tropicales realicen cruzamientos entre

\section{INTRODUCTION}

Cattle producers in tropical regions commonly work towards improving production traits by

Recibido el 20 de enero de 2012. Aceptado el 28 de agosto de 2012.

a Departamento de Genética y Bioestadística, Facultad de Medicina Veterinaria y Zootecnia, Universidad Nacional Autónoma de México. Av. Universidad 3000, Copilco el Alto, 04510 México DF. México. h.toledo.a@gmail.com. Correspondencia al primer autor. 
diferentes razas en forma empírica para mejorar algunas características productivas, utilizando como población base hembras Cebú (Bos indicus) y apareándolas con sementales de razas europeas (Bos taurus), escogidas arbitrariamente y generando eventualmente poblaciones multirraciales. Cualquiera que sea el cruzamiento entre razas, se observará respuesta de vigor híbrido; sin embargo, para mantener niveles aceptables de heterosis en la población, es necesario establecer esquemas de cruzamiento que maximicen la respuesta de las características de interés del productor. Los efectos de cruzamientos entre dos o más razas ha sido ampliamente referido en la literatura(1-12), mostrando las ventajas que se obtienen debido a la heterosis, ya sea individual o materna, para características productivas y reproductivas en ganado de carne(13). La mayor respuesta se alcanza cuando los animales cruzados presentan el $100 \%$ de heterosis, resaltando la importancia de cruzamientos sistemáticos entre las diferentes razas, y explicado como la superioridad de los animales cruzados con respecto a las razas originales. Se ha mostrado que la proporción óptima entre el 50 y $80 \%$ de genes Bos taurus expresan la mayor producción de leche en la cruza Europeo-Cebú en condiciones tropicales(14).

Con base en lo anterior, el objetivo del presente estudio fue determinar la proporción óptima de genes Bos Indicus: Bos taurus y la retención de heterosis, sobre las características de peso al destete ajustado a 207 días, y edad a primer parto en vaquillas cruzadas productoras de carne en una población multirracial en el trópico mexicano.

\section{MATERIALES Y MÉTODOS}

Se utilizaron 1,289 registros de hembras de primer parto con diversas proporciones de genes B. indicus y B. taurus (Charolais, Suizo, Simmental, Holstein Friesian y Salers), tanto animales puros y cruzados; nacidas en el periodo de 1966 a 2006. Los registros se obtuvieron de un rancho comercial ubicado en el municipio crossing different breeds based on empirical criteria. Zebu (Bos indicus) cows are mated with arbitrarily-selected European breed (Bos taurus) bulls, eventually creating multi-breed populations. Hybrid vigor occurs no matter the cross combination, but maintaining acceptable heterosis levels requires crossing systems that maximize response for desired traits. Extensive research into crossbreeding between two or more breeds(1-12) has shown that heterosis, be it individual or maternal, produces benefits for productive and reproductive traits in beef cattle ${ }^{(13)}$. Response is optimum in animals with $100 \%$ heterosis, highlighting the importance of systematic crossbreeding and the superiority of cross-bred animals versus the original breeds. For example, optimum milk production under tropical conditions for European-Zebu crosses is attained with 50 to $80 \%$ Bos taurus genes(14). The present study objective was to determine the optimum Bos I ndicus:Bos taurus gene proportion and retention of heterosis for 207-d adjusted weaning weight and age at first parturition in a multi-breed population of crossbred beef cattle in the tropics of Mexico.

\section{MATERIALS AND METHODS}

Analyses were done of 1,289 records for first parturition cows of various $B$. indicus $x B$. taurus (Charolais, Swiss, Simmental, Holstein, Friesian and Salers) gene proportions, both pure and crossbred, born between 1966 and 2006. Records were from a commercial ranch in Hueytamalco municipality, Puebla, Mexico. Located in the northeast mountains of the state of Puebla, regional climate is humid, semi-hot subtropical (Af[C]), with an annual average temperature of $20.8{ }^{\circ} \mathrm{C}$, a minimum of $15.3^{\circ} \mathrm{C}$ in winter, and a maximum of $24.2{ }^{\circ} \mathrm{C}$ in summer. Documented crosses involved 136 bulls, of which 86 were purebreds (Zebu [16], Charolais [22], Swiss [19], Simmental [10], Holstein [16], Salers [3]), and the remaining 50 were crosses with proportions of these breeds ranging from 6.25 to $75 \%$. Analysis was done of their contributions to the variables 270-d adjusted weaning weight (WW, in kilograms) and age at 
de Hueytamalco, Puebla, México ubicado en la sierra nororiente del estado de Puebla y con clima subtropical húmedo-semicálido Af(c). La temperatura promedio anual es de $20.8^{\circ} \mathrm{C}$, la mínima de $15.3^{\circ} \mathrm{C}$ en invierno, y la máxima es de $24.2{ }^{\circ} \mathrm{C}$ en verano. Se identificó la contribución sobre las variables de peso al destete ajustado a 270 días (PD) y la edad a primer parto (EPP) en días, de 136 diferentes sementales de las razas: Cebú (16), Charolais (22), Suizo (19), Simmental (10), Holstein (16), Salers (3), y de 50 sementales cruzados en diferentes proporciones entre estas razas, desde 6.25 a $75 \%$. Esto ha generado cruzamientos con varios grados de encaste sobre hembras cebú y cruzadas, por lo que el análisis de retención de heterosis o vigor híbrido puede ser identificado de acuerdo a la proporción de la raza materna y paterna.

La heterosis retenida (RVH) se estimó como(15):

$R V H=\left(1-\sum_{i=1}^{n} P s_{i} P m_{i}\right)$

Donde $\mathrm{Ps}_{\mathrm{i}}$ es la proporción de la i-ésima raza del padre; $\mathrm{Pm}_{\mathrm{i}}$ es la proporción de la i-ésima raza de la madre; $n$ es el número total de razas involucradas.

La base de la alimentación fue pastoreo rotacional en potreros con pastos tropicales, manteniendo grupos de hembras de número variable; el apareamiento se realizó con monta natural controlada e inseminación artificial con semen importado de Estados Unidos de América. El criterio de selección de sementales fue arbitrario, ya que el ganadero seleccionaba la raza del toro por gustos personales, esperando que se diera la mejor cruza de acuerdo a la composición racial de las vacas. Por lo que los porcentajes de razas varían desde animales puros hasta animales con la contribución de hasta cinco tipos raciales diferentes.

Se realizó un análisis de regresión múltiple (SASv9; proc reg, selection, rsquare, cp) ${ }^{(16)}$ a first parturition (AFP, in days). Data included crosses of different degrees based on purebred Zebu and crossbred cows; analysis of retention of heterosis (i.e. hybrid vigor) was therefore done by identifying the proportions of the maternal and paternal breeds.

Retained heterosis (RVH) was estimated with the formula(15):

$R V H=\left(1-\sum_{i=1}^{n} P s_{i} P m_{i}\right)$

Where $\mathrm{Ps}_{\mathrm{i}}$ is the proportion of the father's $\mathrm{n}^{\text {th }}$ breed; $\mathrm{Pm}_{\mathrm{i}}$ is the proportion of the mother's $\mathrm{n}^{\text {th }}$ breed; and $\mathrm{n}$ is the total number of breeds involved.

Cows were kept in groups of variable sizes and fed based on rotational grazing on tropical grasses. Mating was done by controlled natural mounting and artificial insemination (semen imported from the United States). Sire selection criteria was arbitrary given that the producer chose bull breeds based on personal preferences with the expectation that an optimum cross would result from cow breed combination. As a result, breed proportions vary from purebreds to individuals with contributions from up to five different breeds.

A multiple regression analysis (SASv9; proc reg, selection, rsquare, cp)(16) was run to identify the effects of breed composition on $\mathrm{WW}$ and AFP. These were based on the Mallow statistic $C(p)$ and the coefficient of determination $\left(R^{2}\right)(17)$ of the relevant crosses between $B$. indicus and the different $\mathrm{B}$. taurus breeds to determine which breeds had significant effects on WW. The analysis was done using a model that included the effects of annual environment (grouped into five categories, each accounting for $20 \%$ of the data between 1966 and 2006) and birth season (April to September and October to March). The percentage of $\mathrm{B}$. indicus genes (C) was included as a covariable to estimate optimum B. indicus: $B$. taurus 
fin de identificar el efecto de las composiciones raciales sobre las características de PD y EPP, con base en el valor del estadístico de Mallow $C(p)$ y del coeficiente de determinación $\left(R^{2}\right)(17)$, de las cruzas relevantes entre $B$. indicus y los distintos tipos raciales de B. Taurus, para poder determinar qué razas tenían un efecto significativo en el peso al destete.

El análisis se realizó con un modelo que incluyó los efectos ambientales de año (agrupándolo en cinco categorías, cada una con el $20 \%$ de los datos desde 1966 hasta el año 2006) y época de nacimiento dependiendo de la época de lluvias (de abril a septiembre y de octubre a marzo), mientras que el porcentaje de genes $B$. indicus (C) se incluyó como covariable, para estimar las proporciones óptimas de $\mathrm{B}$. indicus y B. taurus; así mismo, se generó el mismo modelo usando el valor de RVH (H) como covariable, para estimar el valor de RVH óptimo para PD y EPP de acuerdo al siguiente modelo(18):

$Y_{i j k l}=\mu+A_{i}+E_{j}+b_{1} C_{k}+b_{2} C^{2}{ }_{k}+b_{3} C^{3} k$ $+\mathrm{e}_{\mathrm{ijkl}}$

Donde $Y_{i j k l}$ es la l-ésima observación aleatoria del peso al destete ajustado y/o edad a primer parto; $\mu$ es la media general; $A_{i}$ es el efecto del i-ésimo año; $E_{j}$ es el efecto de la j-ésima época de nacimiento; $C$ es el porcentaje de genes Bos indicus o la heterosis retenida (RVH); $b_{1}, b_{2}, b_{3}$ son los coeficientes de regresión para $\mathrm{C}$, en su forma lineal, cuadrática y cúbica; $\mathrm{e}_{\mathrm{ijkl}}$ el error experimental. $D N I \sim\left(0, \sigma^{2}\right)$.

Con objeto de estimar la mejor proporción de la raza(s) europea(s) con el cebú se utilizó el método stepwise (SAS v9), definiendo el mejor modelo de acuerdo al coeficiente de determinación $\left(R^{2}\right)$ y el estadístico de Mallow; el mejor modelo se graficó con el programa MatLab (con el procedimiento plots) y se obtuvieron el o los puntos de inflexión de acuerdo a la primera derivada (MatLab; roots)(19), que permite estimar a ese punto como los valores óptimos de heterosis para las proportions. The same model was generated using the RVH $(\mathrm{H})$ value as a covariable to the optimum RVH value for $\mathrm{WW}$ and AFP according the model(18):

$Y_{i j k l}=\mu+A_{i}+E_{j}+b_{1} C_{k}+b_{2} C^{2}{ }_{k}+b_{3} C^{3}{ }_{k}$ $+\mathrm{e}_{\mathrm{ijkl}}$

Where $Y_{i j k l}$ is the I-th random observation of adjusted WW and/or AFP; $\mu$ is the general mean; $A_{i}$ is the effect of the $i$-th year; $E_{j}$ is the effect of the $\mathrm{j}$-th birth season; $C$ is the percentage of $\mathrm{B}$. indicus genes or retained heterosis (RVH); $b_{1}, b_{2}, b_{3}$ are the regression coefficients for $\mathrm{C}$ in its linear, quadratic and cubic forms (respectively); and $e_{i j k l}$ is experiment error. $D N I \sim\left(0, \sigma^{2}\right)$.

A stepwise analysis (SAS v9), was run to estimate the optimum proportion of Zebu to European breeds, defining the best model based on the coefficient of determination $\left(R^{2}\right)$ and the Mallow statistic. The best model was graphed using the plots procedure in the MatLab program to generate the inflection points according to the first derivative (MatLab; roots)(19). This allowed estimation of this point as the optimum heterosis values for $\mathrm{WW}$ and AFP in $\mathrm{B}$. indicus $\times \mathrm{B}$. taurus crosses.

\section{RESULTS}

No differences $(P>0.05)$ were identified in the analysis of variance between the Zebu and European breeds, or the crosses, for either of the studied variables (Table 1). This absence of difference is most probably due to very high variation in the Zebu breed compared to other breeds; for instance, compared to European breeds it is five times more variable in terms of WW and three times more variable for AFP, while compared to crosses it is eleven times more variable. Indeed, crosses and European breeds tend to be much more homogeneous in their responses.

Selection for the best model showed the Zebu $x$ Simmental cross to contribute most $(P<0.05)$ 
EFECTO DE LA PROPORCIÓN DE GENES SOBRE CARACTERÍSTICAS DE PESO AL DESTETE

Cuadro 1. Promedios, desviaciones estándar (S) y coeficientes de variación (CV) para peso al destete ajustado a 270 días y edad al primer parto en vacas Cebú, Bos Taurus (todos los animales de raza pura europea), Charolais, Holstein, Simmental, Suizo y cruzas entre dos o más razas

Table 1. Averages, standard deviations (S) and coefficients of variation (CV) for 270-d adjusted weaning weight and age at first parturition in Zebu, Bos Taurus (all European purebreds), Charolais, Holstein, Simmental, Swiss and crosses between two or more breeds

\begin{tabular}{|c|c|c|c|c|}
\hline \multirow[b]{2}{*}{ Breed } & \multicolumn{2}{|c|}{ Weaning weight } & \multicolumn{2}{|c|}{ Age at first parturition } \\
\hline & $\bar{X} \pm S$ & CV (\%) & $\bar{X} \pm S$ & CV (\%) \\
\hline Zebu & $209.85 \pm 20.69$ & 9.85 & $1109.55 \pm 99.35$ & 8.95 \\
\hline Bos Taurus & $222.52 \pm 4.73$ & 2.12 & $1100.48 \pm 29.83$ & 2.71 \\
\hline Charolais & $253.16 \pm 13.09$ & 5.17 & $1181.59 \pm 74.51$ & 6.30 \\
\hline Holstein & $228.79 \pm 18.51$ & 8.09 & $914.82 \pm 112.65$ & 12.31 \\
\hline Simmental & $213.83 \pm 6.38$ & 2.98 & $1123.61 \pm 40.94$ & 3.64 \\
\hline Swiss & $223.89 \pm 9.25$ & 4.13 & $1049.47 \pm 60.84$ & 5.79 \\
\hline Crosses & $225.52 \pm 4.73$ & 2.09 & $1099.00 \pm 9.01$ & 0.82 \\
\hline
\end{tabular}

características PD y EPP en la cruza de B. indicus con B. taurus.

\section{RESULTADOS}

El Cuadro 1 muestra los promedios, desviaciones estándar (S) y coeficientes de variación (CV) para peso al destete ajustado a 270 días y edad al primer parto en vacas Cebú, Bos Taurus (todos los animales de raza pura europea), Charolais, Holstein, Simmental, Suizo y Cruzadas entre dos o más razas. Si bien el análisis de varianza no mostró diferencias $(P>0.05)$ entre la raza cebú con las europeas y las cruzas en ninguna de las variables estudiadas, esto puede deberse a que la variación observada en la raza cebú es cinco veces mayor en peso al destete y tres veces mayor en edad al primer parto con respecto a las razas europeas, así como 11 veces mayor con respecto a las vacas cruzadas, siendo los animales cruzados y europeos más homogéneos en su respuesta.

En el Cuadro 2 se presentan los coeficientes de determinación, los estadísticos de Mallow y el error estándar para la selección del mejor modelo a partir del análisis de regresión múltiple, to explaining the variation, both for WW (46.13\%) and AFP (9.09\%) (Table 2). For crosses of three or more breeds, contributions by Salers and Charolais sires increased WW by 12 and $4 \%$, respectively, while four-breed crosses increased this variable by $12.57 \%$. In contrast, AFP increased less than $1 \%$ in crosses of three or more breeds.

The WW coefficient of determination results for the linear, quadratic and cubic models showed the different European breeds to contribute equally when crossed with $\mathrm{B}$. indicus (Table 3 ). For WW, the quadratic model explained $23.8 \%$ $(\mathrm{P}<0.01)$ of variation, suggesting the presence of complementarity between Zebu and the European breeds. When three or more breeds were combined, this heterosis increased; such is the case for the crosses Zebu $x$ Charolais $x$ Swiss $\left(R^{2}=0.1469 ; P<0.0051\right)$; Zebu $x$ Charolais $x$ Simmental $\left(R^{2}=0.3016\right.$; $\left.P<0.0001\right)$; Zebu $x$ Charolais $x$ Simmental $x$ Salers $\left(R^{2}=0.3225\right.$ $\mathrm{P}<0.0001)$; and Zebu $\mathrm{x}$ Charolais $\mathrm{x}$ Swiss $\mathrm{X}$ Simmental $x$ Salers $\left(R^{2}=0.2411 ; P<0.0001\right)$.

The coefficients of determination and significance levels for AFP identified complementarity between $\mathrm{B}$. indicus and $\mathrm{B}$. 
Hugo O. Toledo Alvarado, et al. / Rev Mex Cienc Pecu 2015;6(1):1-13

Cuadro 2. Análisis de regresión para la selección de los modelos para peso al destete (ww) y edad al primer parto (AFP) $(P<0.05)$

Table 2. Regression analysis for model selection $(P<0.05)$ for $270-d$ adjusted weaning weight (WW) and age at first parturition (AFP)

\begin{tabular}{|c|c|c|c|}
\hline Breeds & $\mathrm{R}^{2}$ & $\mathrm{C}_{(\mathrm{p})}$ & SE \\
\hline \multicolumn{4}{|l|}{ WW } \\
\hline Zebu x Charolais & 0.0505 & 40.51 & 6970.89 \\
\hline Zebu $x$ Swiss & 0.0300 & 41.45 & 7121.45 \\
\hline Zebu x Simmental & 0.4613 & 21.69 & 3955.21 \\
\hline Zebu $x$ Charolais $\times$ Swiss & 0.5739 & 18.52 & 3753.85 \\
\hline Zebu x Simmental x Salers & 0.5809 & 18.20 & 3692.08 \\
\hline Zebu $\times$ Charolais $\times$ Simmental & 0.5017 & 21.83 & 4390.25 \\
\hline Zebu $\times$ Charolais $\times$ Simmental $\times$ Salers & 0.5870 & 19.92 & 4547.78 \\
\hline Zebu $x$ Charolais $x$ Swiss $\times$ Simmental $\times$ Salers & 0.9345 & 6.00 & 961.14 \\
\hline \multicolumn{4}{|l|}{ AFP } \\
\hline Zebu x Holstein & 0.0873 & 1.29 & 37640 \\
\hline Zebu x Simmental & 0.0909 & 1.13 & 37490 \\
\hline Zebu x Charolais $\times$ Holstein & 0.0930 & 3.04 & 38319 \\
\hline Zebu x Simmental x Holstein & 0.0938 & 3.01 & 38284 \\
\hline Zebu $\times$ Charolais $\times$ Swiss $\times$ Simmental & 0.0940 & 5.00 & 39231 \\
\hline Zebu $x$ Charolais $\times$ Swiss $\times$ Simmental $\times$ Holstein & 0.0940 & 4.99 & 39231 \\
\hline
\end{tabular}

$R^{2}=$ coefficient of determination, $C_{(\mathrm{p})}=$ Mallow statistic, $\mathrm{SE}=$ standard error.

Significant results used in figures are indicated in bold.

mostrando la contribución del cruzamiento de dos, tres o más razas $(\mathrm{P}<0.05)$ sobre las variables estudiadas; estos resultados revelaron que el cruce con las razas Cebú y Simmental son las que mayor contribuyen en la explicación de la variación, tanto de peso al destete ajustado, como de edad al primer parto, siendo 46.13 y $9.09 \%$ respectivamente.

En el análisis de cruces con tres o más razas se observó que para peso ajustado al destete, la contribución de sementales de raza Salers o Charolais incrementan la respuesta en 12 y $4 \%$ respectivamente, mientras que el uso de cuatro razas incrementa en $12.57 \%$ el peso al destete. Sin embargo, con respecto a edad al primer parto, sólo se observó un incremento menor al $1 \%$ con la cruzas de tres o más razas.

En el Cuadro 3 se presentan los coeficientes de determinación de los modelos lineal, cuadrático taurus genes as expressed in the quadratic model $\left(R^{2}=0.1843 ; P<0.0001\right)$. The best heterosis response occurred in the Zebu $\mathrm{X}$ Simmental cross $\left(R^{2}=0.4796 ; P<0.0334\right)$.

270-day adjusted weaning weight (WW)

The contribution of $\mathrm{B}$. indicus based on the quadratic model $\left(R^{2}=0.238 ; P<0.01\right.$; Figure 1$)$, produced the greatest heterosis response at a $50.22 \%$ Zebu allele proportion, which resulted in a $\mathrm{WW}$ of $239 \mathrm{~kg}$. However, this assumes an equal contribution of all the European breeds to $\mathrm{WW}$, with each breed potentially contributing positively or negatively.

When analyzed by cross (Table 5 ), the quadratic model $\left(R^{2}=0.1469 ; P<0.05\right)$ showed the $B$. indicus $x$ Charolais $x$ Swiss cross to have the lowest response in WW (220.13 kg) with $36.5 \%$ $B$. indicus genes. In contrast, the $B$. indicus $x$ 
EFECTO DE LA PROPORCIÓN DE GENES SOBRE CARACTERÍSTICAS DE PESO AL DESTETE

Cuadro 3. Coeficiente de determinación $\left(R^{2}\right)$ para cada modelo de peso al destete ajustado a 270 días Table 3. Coefficient of determination $\left(R^{2}\right)$ for each $270-d$ adjusted weaning weight model

\begin{tabular}{lcccccc}
\hline & Linear & $P<$ & Quadratic & $P<$ & Cubic & $P<$ \\
\hline B. indicus x B. taurus & 0.2338 & 0.0001 & $\mathbf{0 . 2 3 8 0}$ & 0.0001 & 0.2384 & 0.0001 \\
Charolais & 0.2659 & 0.1471 & 0.3165 & 0.1310 & 0.3346 & 0.1708 \\
Swiss & 0.1666 & 0.1321 & 0.1675 & 0.2085 & 0.1694 & 0.2961 \\
Simmental & 0.1516 & 0.7079 & 0.1518 & 0.8151 & 0.1535 & 0.8880 \\
Charolais- Swiss & 0.1449 & 0.0027 & $\mathbf{0 . 1 4 6 9}$ & 0.0051 & 0.1470 & 0.0096 \\
Simmental-Salers & 0.3025 & 0.0743 & 0.3090 & 0.1147 & 0.3529 & 0.0984 \\
Charolais-Simmental & 0.2972 & 0.0001 & $\mathbf{0 . 3 0 1 6}$ & 0.0001 & 0.3026 & 0.0001 \\
Charolais-Simmental-Salers & 0.3184 & 0.0001 & $\mathbf{0 . 3 2 2 5}$ & 0.0001 & 0.3229 & 0.0001 \\
Charolais-P.S.-Simmental-Salers & 0.2368 & 0.0001 & $\mathbf{0 . 2 4 1 1}$ & 0.0001 & 0.2415 & 0.0001 \\
\hline
\end{tabular}

$P=$ significance level. Significant results used in figures are indicated in bold.

Cuadro 4. Coeficiente de determinación $\left(R^{2}\right)$ para cada modelo de edad a primer parto

Table 4. Coefficient of determination $\left(R^{2}\right)$ for each age at first parturition model

\begin{tabular}{lcccccc}
\hline & Linear & $P<$ & Quadratic & $P<$ & Cubic & $P<$ \\
\hline B. indicus x B. taurus & 0.1835 & 0.0001 & $\mathbf{0 . 1 8 4 3}$ & 0.0001 & 0.1845 & 0.0001 \\
Simmental & 0.4376 & 0.0328 & $\mathbf{0 . 4 7 9 6}$ & 0.0334 & 0.5192 & 0.0342 \\
Holstein & 0.0999 & 0.8777 & 0.1083 & 0.9541 & 0.1083 & 0.9541 \\
Simmental-Holstein & 0.1918 & 0.1655 & 0.1932 & 0.2450 & 0.2129 & 0.2630 \\
Charolais-Holstein & 0.0753 & 0.5125 & 0.0756 & 0.6325 & 0.0803 & 0.7004 \\
Charolais-P.S.-Simmental & 0.1781 & 0.0001 & $\mathbf{0 . 1 7 8 3}$ & 0.0001 & 0.1783 & 0.0001 \\
\hline
\end{tabular}

$P=$ significance level. Significant results used in figures are indicated in bold.

y cúbico de las cruzas entre $B$. indicus con razas europeas para PD ajustado a 270 días; en la cruza con B. taurus, se considera igual la contribución de todas las razas europeas analizadas en este estudio, observando que el modelo cuadrático explicó el $23.8 \%$ ( $\mathrm{P}<0.01)$; este resultado sugiere que existe una complementariedad de los genes Cebú y razas europeas para peso al destete. Esta heterosis, es mayor cuando existe la combinación de tres o más razas, tal es el caso de Cebú x Charolais $x$ Suizo $\left(R^{2}=0.1469 ; \quad P<0.0051\right)$; Cebú $x$ Charolais $\times$ Simmental $\left(R^{2}=0.3016 ; P<0.0001\right)$; Cebú $\mathrm{x}$ Charolais $\mathrm{x}$ Simmental $\mathrm{x}$ Salers $\left(R^{2}=0.3225 P<0.0001\right)$ y Cebú $x$ Charolais $x$ Suizo $\times$ Simmental $\times$ Salers $\left(R^{2}=0.2411\right.$; $\mathrm{P}<0.0001)$.
Charolais $\times$ Simmental cross had the highest response in WW $(245.13 \mathrm{~kg})$ with $70.75 \% \mathrm{~B}$. indicus genes, as explained by the quadratic model $\left(R^{2}=0.3016 ; P<0.0001\right)$. For a cross between four breeds (B. indicus $x$ Charolais $x$ Simmental $x$ Salers), the quadratic model $\left(R^{2}=0.3225\right.$; $\mathrm{P}<0.0001)$ showed an optimum response $(\mathrm{WW}=$ $243.72 \mathrm{~kg}$ ) at $66.5 \%$ B. indicus genes. Therefore, optimum heterosis response was had with 36.5 to $70.75 \% \mathrm{~B}$. indicus genes in combinations of three to four breeds; the highest WW was observed with the $B$. indicus $x$ Charolais $x$ Simmental cross. Complementarity between $B$. indicus and $\mathrm{B}$. taurus can largely explain this improved WW since $B$. indicus genes contribute greater environmental adaptability while B. taurus genes provide greater growth capacity. 
Hugo O. Toledo Alvarado, et al. / Rev Mex Cienc Pecu 2015;6(1):1-13

Con respecto a edad a primer parto, los coeficientes de determinación y significancia se muestran en el Cuadro 4. Estos resultados revelan que existe complementariedad entre los genes $\mathrm{B}$. indicus con $\mathrm{B}$. taurus expresado por el modelo cuadrático ( $R^{2}=0.1843$; $\left.P<0.0001\right)$. Sin embargo, la mayor respuesta a heterosis se observó en la cruza de Cebú x Simmental $\left(R^{2}=0.4796 ; P<0.0334\right)$.

Peso al destete ajustado a 270 días (PD)

La respuesta a heterosis estimada a partir de la contribución de $B$. indicus, se muestra en el la Figura 1 a partir del modelo cuadrático $\left(R^{2}=0.238 ; P<0.01\right)$. En estos resultados se observa que la mayor respuesta de heterosis se alcanza cuando la proporción de alelos cebuinos se encuentra cercano a $50.22 \%$, siendo el PD de $239 \mathrm{~kg}$. Sin embargo, estos resultados suponen que el complemento de todas las razas europeas contribuye igualmente en la característica, siendo que cada raza puede contribuir positiva o negativamente.

En el Cuadro 5 se presenta el análisis por cruza; la cruza de $B$. indicus $\times$ Charolais $\times$ Suizo tuvo la menor respuesta de PD cuando existe un $36.5 \%$ de genes B. indicus con $220.13 \mathrm{~kg}$, explicado por el modelo cuadrático $\left(R^{2}=0.1469\right.$; $P<0.05)$. Así mismo la cruza de $B$. indicus $x$ Charolais $\times$ Simmental, tiene el mayor PD cuando tiene el $70.75 \%$ de genes $B$. indicus y está expresado por el modelo cuadrático $\left(R^{2}=0.3016\right.$; $P<0.0001)$ con un PD de $245.13 \mathrm{~kg}$. La cruza de cuatro razas: $B$. indicus $x$ Charolais $x$ Simmental $x$ Salers, está justificada por el modelo cuadrático $\left(\mathrm{R}^{2}=0.3225 ; \mathrm{P}<0.0001\right)$ con un valor óptimo de genes $B$. indicus de $66.5 \%$ y con un peso de $243.72 \mathrm{~kg}$. Por lo anterior se puede ubicar la mejor respuesta a heterosis dentro del rango de 36.5 a $70.75 \%$ de genes B. indicus en la contribución de 3 y 4 razas, teniendo el mayor peso en la cruza B. indicus $x$ Charolais $x$ Simmental. Esto puede ser explicado por una mejor adaptabilidad al medio ambiente debido a la complementariedad de las razas involucradas, dado que los genes $B$.
Figura 1. Respuesta a heterosis en la cruza de Bos indicus $\times$ Bos taurus para para peso al destete ajustado a 270 días (WW) y para edad a primer parto (AFP)

Figure 1. Response to heterosis in Bos indicus x Bos taurus crosses for 270-d adjusted weaning weight (WW) and age at first parturition (AFP)
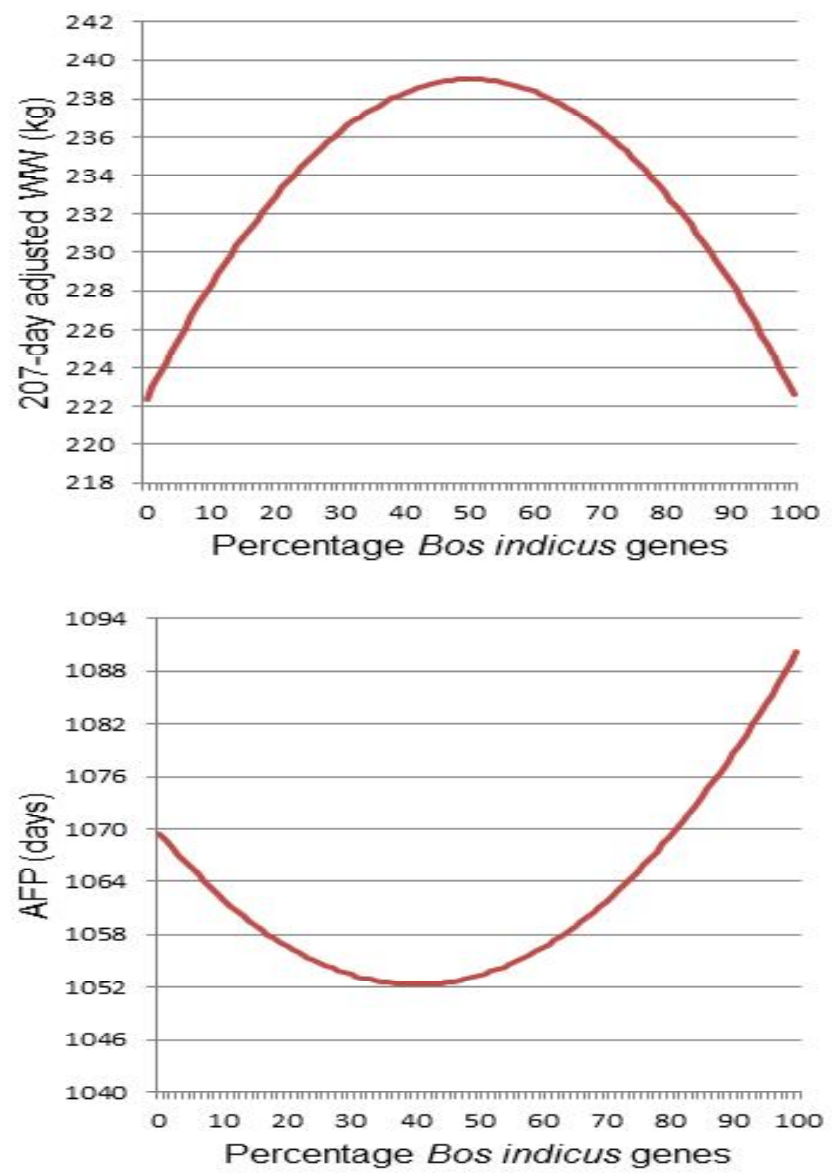

In the five-breed cross (B. indicus $x$ Charolais $x$ Swiss $x$ Simmental $x$ Salers), optimum response $(\mathrm{WW}=236.08 \mathrm{~kg})$ according to the quadratic model $\left(R^{2}=0.2411 ; P<0.0001\right)$ was observed with $49.94 \% \mathrm{~B}$. indicus genes. It is of note that this proportion is nearly that of a basic $\mathrm{B}$. indicus $\times \mathrm{B}$. taurus cross $(50 \% \mathrm{~B}$. indicus genes), and that the response was lower than for a Charolais $x$ Simmental cross. This is consistent with previous reports showing that crosses between Angus, Hereford and Shorthorn breeds exhibit up to an $\mathbf{1 8 . 9} \%$ better response in WW than purebred animals(4). 
indicus contribuye con una mayor adaptabilidad al medio ambiente y los genes $\mathrm{B}$. taurus aportan una mejor capacidad de crecimiento. Así mismo, en la cruza de cinco razas: $B$. indicus $x$ Charolais $x$ Suizo x Simmental $x$ Salers, se obtuvo la mejor respuesta con un porcentaje de genes $\mathrm{B}$. indicus de $49.94 \%$, con un peso de $236.08 \mathrm{~kg}$ y es explicada por el modelo cuadrático $\left(R^{2}=0.2411 ; P<0.0001\right)$, sin embargo, es menor la respuesta que en la cruza con Charolais y Simmental. La cruza de cinco razas muestra una relación similar a la presentada para la cruza B. indicus x B. taurus cercana al $50 \%$ de genes $B$. indicus. Se ha estimado que las cruzas entre Angus, Hereford y Shorthorn tuvieron una superioridad de hasta $18.9 \%$ de animales con cruzados sobre los animales de razas puras(4).

Edad a primer parto (EPP)

La respuesta a heterosis para EPP en la cruza $B$. indicus $\times$ B. taurus, se puede ver en la Figura 1; el efecto del porcentaje racial de origen
Age at first parturition (AFP)

For AFP, the best heterosis response (average $=$ $1052.31 \mathrm{~d}$ ) for the overall $B$. indicus $\times$ B. taurus cross was shown by the quadratic model $\left(R^{2}=0.1843 ; P<0.0001\right)$, with a $40.20 \% B$. indicus gene proportion (Figure 1 ). The $\mathrm{B}$. indicus $x$ Simmental cross had the best response (1083.41 d AFP) with $30.68 \%$ B. indicus genes, according to the quadratic model $\left(R^{2}=0.4796\right.$; $\mathrm{P}=0.0334$; Table 5). These results confirm the advantages of using $B$. indicus $\times$ B. taurus crosses to improve cattle reproductive traits, and agree with a report of AFP in crosses between Angus, Hereford and Shorthorn, that showed these to have younger AFP than purebreds(4). This is supported by another report indicating the superiority of crosses (Angus, Brahman, Brangus and Hereford) over purebreds in other reproductive traits such as calves born and weaned calves(1). In a study of purebred Criollo and Guzerat cows and their F1 crosses, estimated individual heterosis and

Cuadro 5. Máxima respuesta a heterosis (MRH) de acuerdo a los modelos para peso al destete ajustado a 270 días (WW), edad a primer parto (AFP) y para la retención de heterosis (RVH)

Table 5. Maximum heterosis response (MHR) based on 270-d adjusted weaning weight (WW) models, age at first parturition (AFP) models and retention of heterosis (RVH)

\begin{tabular}{llll}
\hline & Equation & MHR (\%) \\
\hline WW & & & \\
B. indicus x B. taurus & $W W=222.3608+0.6629^{*} C-0.0066^{*} C^{2}$ & 50.22 & $239.00 \mathrm{~kg}$ \\
Charolais-Simmental & $W W=214.7215+0.8596^{*} C-0.0061^{*} C^{2}$ & 70.75 & $245.13 \mathrm{~kg}$ \\
Charolais-Swiss & $W W=216.1266+0.2191^{*} C-0.0029^{*} C^{2}$ & 36.50 & $220.13 \mathrm{~kg}$ \\
Charolais-Simmental-Salers & $W W=216.6918+0.8010^{*} C-0.006^{*} C^{2}$ & 66.50 & $243.72 \mathrm{~kg}$ \\
Charolais-Swiss-Simmental-Salers & $W W=220.0803+0.64^{*} C-0.0064^{*} C^{2}$ & 49.94 & $236.08 \mathrm{~kg}$ \\
AFP & & & \\
Bos indicus x Bos taurus & $A F P=1069.46-0.8531^{*} C+0.0106^{*} C^{2}$ & 40.201052 .31 days \\
Simmental & $A F P=1218.72-8.8190^{*} C+0.1436^{*} C^{2}$ & 30.681083 .41 days \\
RVH & & & \\
Quadratic & $W W=209.2959+55.59^{*} H-36.36^{*} H^{2}$ & 76 & $232.58 \mathrm{~kg}$ \\
Cubic & $W W=215.33+5.42^{*} H+70.65^{*} H^{2}-63.12^{*} H^{3}$ & 78 & \\
Quadratic & $A F P=1070.26-59.9331^{*} H+32.5085^{*} H^{2}$ & 92 & 1023.33 days \\
Cubic & $A F P=1045.57+236.38^{*} H-699.24^{*} H^{2}+461.27^{*} H^{3}$ & 79 & \\
\hline
\end{tabular}

WW and AFP model equations: $C$ represents percentage of $B$. indicus genes. RVH equations: $H$ represents retention of heterosis. 
cebuino, fue representado por el modelo cuadrático $\left(R^{2}=0.1843 ; P<0.0001\right)$ teniendo la mejor respuesta de EPP cuando el porcentaje de genes B. indicus se ubicó en $40.20 \%$ con un promedio de 1052.31 días.

La cruza de $B$. indicus $x$ Simmental se explica por el modelo cuadrático que se encuentra en el Cuadro $5\left(R^{2}=0.4796 ; P=0.0334\right.$, ) teniendo la mejor respuesta a heterosis con un $30.68 \%$ de genes cebuínos con 1083.41 días de EPP. Diversos estudios mencionan la importancia de utilizar animales cruzados $\mathrm{B}$. indicus $\times \mathrm{B}$. Taurus para mejorar las características reproductivas. Se ha reportado que animales con cruzas entre Angus, Hereford y Shorthorn tuvieron una EPP inferior a animales de razas puras, en concordancia con los resultados de este estudio(4). Así mismo en otro estudio, explicaron la superioridad de animales cruzados (Angus, Brahman, Brangus y Herford) sobre animales de razas puras en otras características reproductivas, como becerros nacidos y becerros destetados(1). También en otra publicación, estimaron la heterosis individual y compararon las tasas de estro, gestación, parición y destete para vacas Criollo, Guzerat y sus crías F1, concluyendo la viabilidad de las cruzas para aprovechar el efecto de la heterosis y mejorar la fertilidad(12).

\section{Heterosis retenida (RVH)}

Debido al origen de los datos, característico de las ganaderías productoras de carne en sistemas extensivos en el trópico, el uso de cruzamientos donde se incluyen varias razas, hace que exista una gran variación racial de los animales; por lo que la estimación de heterosis retenida ( $\mathrm{RVH}$ ) permite establecer el comportamiento fenotípico en función de la contribución de los progenitores, ya sean puros o cruzados.

Con respecto al PD, se observó un incremento a medida que el grado de heterosis fue aumentando, alcanzando la mayor respuesta en los niveles de 76 a $78 \%$ de retención de heterosis distribuido en diferentes razas. Esto comparisons of estrus, gestation, parturition and weaning highlighted the advantages of heterosis in the crosses to improve fertility(12).

Retained heterosis ( $\mathrm{RVH})$

The data analyzed in the present study are characteristic of beef cattle producers in extensive tropical conditions. Use of crosses involving a number of different breeds results in wide genetic variation. Estimation of $\mathrm{RVH}$ can establish phenotypic behavior as a function of progenitor contribution, be they purebred or crosses. As degree of heterosis increased so did $\mathrm{WW}$, reaching levels of $76-78 \% \mathrm{RVH}$ among the different breeds (Table 5). For every RVH unit, WW increased $0.276 \mathrm{~kg}$, reaching a maximum of $232.58 \mathrm{~kg}$. This was explained by both the quadratic $\left(R^{2}=0.2370 ; P<0.0001\right)$ and cubic $\left(R^{2}=0.2375 P<0.0001\right)$ models. For AFP, maximum RVH occurred between 79 and $92 \%$, with a minimum value of $1023.33 \mathrm{~d}$; this was explained by the quadratic $\left(R^{2}=0.1792 ; P<0.0001\right)$ and cubic $\left(R^{2}=0.1813 ; P<0.0001\right)$ models.

In a comparison of $\mathrm{RVH}$ values for $\mathrm{WW}$ and AFP, and their corresponding averages (Table 6), the highest WW was expressed with an approximately $80 \% \mathrm{RVH}$, both for the quadratic $(230.49 \mathrm{~kg})$ and cubic $(232.56 \mathrm{~kg})$ models. The lowest AFP occurred at $90 \% \mathrm{RVH}$ in the quadratic model $(1,042.65 \mathrm{~d})$ and at $80 \%$ $\mathrm{RVH}$ in the cubic model $(1,023.33 \mathrm{~d})$. Therefore, the best heterosis response is attained at between 80 and $90 \% \mathrm{RVH}$.

\section{DISCUSSION}

Animal growth directly affects productive performance, including WW and AFP. Heterosis has a well-documented beneficial effect on productive traits, indeed, it is popularly known as hybrid vigor. The $\mathrm{B}$. indicus $\times \mathrm{B}$. taurus cross is widely used for its complementarity, with proportions quantified as fractions of the breeds involved. The most frequently used fraction is $3 / 8 \mathrm{~B}$. indicus - $5 / 8 \mathrm{~B}$. taurus, which is known to have good performance. However, in the 
se muestra en el Cuadro 5, observando un incremento de $0.276 \mathrm{~kg}$ por cada unidad de retención de heterosis, y es explicado por el modelo cuadrático $\left(R^{2}=0.2370 ; P<0.0001\right)$ y por el modelo cúbico $\left(\mathrm{R}^{2}=0.2375 \mathrm{P}<0.0001\right)$ con un peso al destete ajustado máximo de $232.58 \mathrm{~kg}$.

También se observa la retención de heterosis óptima para tener una menor EPP y fue representada por el modelo cuadrático $\left(R^{2}=0.1792 ; P<0.0001\right)$ y por el modelo cúbico $\left(R^{2}=0.1813 ; P<0.0001\right)$ ubicando la proporción de retención de heterosis óptima entre un 79 y un $92 \%$ con un valor mínimo de 1023.33 días de edad a primer parto. En el Cuadro 6 se muestran los diferentes porcentajes de retención de heterosis con sus respectivos promedios para el PD y la EPP. El mayor PD se expresó con una RVH alrededor del $80 \%$, tanto para el modelo cuadrático $(230.49 \mathrm{~kg})$ como para el modelo cúbico $(232.56 \mathrm{~kg})$. La menor EPP se obtuvo con una RVH del $90 \%$ en el modelo cuadrático con $1,042.65$ días y con una RVH del $80 \%$ en el modelo cúbico con 1,023.33 días. Esto explica que la mejor respuesta a heterosis se alcanza con una RVH entre el 80 y $90 \%$.

\section{DISCUSIÓN}

El crecimiento de un animal impacta directamente sobre el desempeño de éste sobre todo el proceso productivo, influyendo en características como el PD y la EPP. Se ha discutido en varias publicaciones, el efecto benéfico que tiene la heterosis sobre las características productivas, llegando a ser muy popular y conocido como vigor hibrido. Para este fin, con más frecuencia se utilizan cruzas entre ganado $\mathrm{B}$. indicus y $\mathrm{B}$. taurus; las proporciones, por lo regular, se manejan en fracciones.

La proporción más frecuentemente usada es la de $3 / 8 \mathrm{~B}$. indicus - 5/8 B. taurus, teniendo buen comportamiento; sin embargo, los resultados muestran que la heterosis se manifiesta en un rango de 42 al $70 \%$ de genes $B$. indicus para
Cuadro 6. Retención de heterosis (RVH) para peso al destete (WW) y edad al primer parto (AFP)

Table 6. Retention of heterosis (RVH) for 270-d adjusted weaning weight (WW) and age at first parturition (AFP)

\begin{tabular}{lccccc}
\hline RVH & \multicolumn{2}{c}{ WW } & & \multicolumn{2}{c}{ AFP } \\
\cline { 2 - 3 } \cline { 5 - 6 }$\%$ & Quadratic & Cubic & & Quadratic & Cubic \\
\hline 0 & 209.29 & 215.33 & & 1070.26 & 1045.57 \\
10 & 214.49 & 216.51 & & 1064.59 & 1062.67 \\
20 & 218.95 & 218.73 & & 1059.57 & 1068.56 \\
30 & 222.70 & 221.61 & & 1055.20 & 1066.00 \\
40 & 225.71 & 224.76 & & 1051.48 & 1057.76 \\
50 & 228.00 & 227.81 & & 1048.42 & 1046.60 \\
60 & 229.56 & 230.38 & & 1046.00 & 1035.30 \\
70 & 230.39 & 232.09 & & 1044.23 & 1026.62 \\
80 & 230.49 & 232.56 & & 1043.11 & 1023.33 \\
90 & 229.87 & 231.42 & & 1042.65 & 1028.19 \\
100 & 228.52 & 228.28 & & 1042.83 & 1043.98 \\
\hline Averagnnnnnn
\end{tabular}

Averages for WW and AFP quadratic and cubic models calculated for different $\mathrm{RVH}$ percentages.

present WW results heterosis was expressed in a 42 to $79 \%$ range of $B$. indicus genes; this is similar to fractions of $2 / 5$ to $3 / 5 \mathrm{~B}$. indicus. In other words, it is roughly equivalent to the hybrid vigor to be expected in an F1 hybrid (i.e. $50 \%$ of each breed).

For AFP, the best response $(1,051 \mathrm{~d})$ was produced with 30 to $40 \% \mathrm{~B}$. indicus genes. This is roughly equivalent to fractions of $3 / 10$ $\mathrm{B}$. indicus and $7 / 10 \mathrm{~B}$. Taurus, a proportion slightly nearer the common $3 / 8 \mathrm{~B}$. indicus to 5/8 B. taurus proportion.

Retention of heterosis manifested the highest potential for WW at 76 to $78 \%$ and for AFP at 79 to $92 \%$. This confirms that reproductive trait responses improve as heterosis retention increases, in agreement with the relevant literature.

\section{CONCLUSIONS AND IMPLICATIONS}

Bos taurus $x$ Bos indicus crosses effectively take advantage of hybrid vigor, improving weaning 
PD; es decir aproximadamente $2 / 5$ a $3 / 5$ de $\mathrm{B}$. indicus aproximadamente, que bien puede considerarse como al efecto esperado de vigor hibrido en la $\mathrm{F}_{1}$ (50\% de cada raza).

Para la característica reproductiva EPP se puede concluir que la mejor respuesta a la heterosis para tener una menor edad a primer parto la encontramos con una proporción de genes cebuínos que va del 30 al $40 \%$, teniendo la menor edad con 1,051 días, siendo esto aproximadamente $3 / 10$ de $B$. indicus y $7 / 10$ de B. Taurus, aproximándose un poco más de la relación comúnmente usada de 3/8 B. indicus y $5 / 8$ B. taurus.

La retención de heterosis que mostró mayor potencial para PD fue de 76 a $78 \%$ y para EPP de 79 a $92 \%$, indicando que entre mayor retención de heterosis, se tiene una mejor respuesta que se expresa para las características productivas, concordando con lo que refiere la bibliografía.

\section{CONCLUSIONES E IMPLICACIONES}

Las cruzas entre razas Bos taurus con Bos indicus se pueden utilizar para aprovechar el vigor hibrido, aumentando las características de peso al destete y de edad a primer parto gracias a la complementariedad de las razas, siempre y cuando la proporción de genes Bos indicus respecto a Bos taurus oscile entre 30 al $40 \%$ para EPP, mientras que para el PD la proporción óptima de genes Bos indicus se encuentra en el rango de 42 al $70 \%$, por lo que es útil en producciones donde se utilicen como animales terminales, o en donde se tiene establecido un sistema de cruzamientos adecuado.

\section{LITERATURA CITADA}

1. Turner JW, Farthing BR, Robertson GL. Heterosis in reproductive performance of beef cows. J Anim Sci 1968; 27:336-338

2. Dickerson GE. Experimental approaches in utilizing breed resources. Anim Breed Abstr 1969;37:191-202. weight and age at first parturition due to breed complementarity. To obtain these responses, Bos indicus: Bos taurus proportions need to range from 30 to $40 \%$ for AFP and from 42 to $70 \%$ for WW. These proportions are useful in production systems involving terminal animals or using an adequate genetic improvement system.

End of english version

3. Koger M, Cunha TJ, Warnick AC editors. Crossbreeding beef cattle. Series 2. Florida, USA: University of Florida Press; 1973.

4. Cundiff LV, Gregory KE, Koch RM. Effects of heterosis on reproduction in Hereford, Angus and Shorthorn Cattle. J Anim Sci 1974; 38:711-727.

5. Franke DE. Breed and heterosis effects of American Zebu cattle. J Anim Sci 1980;50:1206-1214.

6. Plasse D. Crossbreeding results from beef cattle in the Latin American tropics. Anim Breed Abstr 1983;51(11):779-797.

7. Plasse D. Estrategias de cruzamiento en ganado de carne. II Cursillo sobre Bovinos de Carne. UCV, Facultad de Ciencias Veterinarias. Maracay, Venezuela. 1986.

8. Koch RM, Dickerson GE, Cundiff LV, Gregory KE. Heterosis retained in advanced generations of crosses among Angus and Hereford cattle. J Anim Sci 1985;60(5):1117-1131.

9. Romero R, Plasse D, Camaripano L. Producción de vacas F1 (Bos taurus $x$ Bos indicus) apareadas con toros cruzados en un hato al sur de Barinas. UCV, Facultad de Ciencias Veterinarias. Barinas, Venezuela, 1997.

10. Chirinos Z, Rincón E, Madrid-Bury N, González-Stagnaro C. Crecimiento predestete de becerros mestizos Bos taurus $x$ Bos indicus. Arch Latinoam Prod Anim 1997; 5(Supl 1) 497499.

11. Rosales AJ, Elzo MA, Montaño BM, Vega MV, Reyes VA. Parámetros genéticos para pesos al nacimiento y destete en ganado Simmental - Brahman en el subtrópico mexicano. Téc Pecu Méx. 2004;42(3):333-346.

12. Martínez VG, Montaño BM, Palacios FJA. Efectos genéticos directos, maternos y heterosis individual para tasas de estro, gestación, parición y destete de vacas Criollo, Guzerat y sus cruzas F1. Téc Pecu Méx 2006;44(2):143-154.

13. Crockett JR, Koger M, Franke DE. Rotational crossbreeding of beef cattle: preweaning traits by generation. J Anim Sci 1978; 46: 1170-1177.

14. López OR, García CR, García MJ G, Ramírez VR. Producción de leche de vacas con diferente porcentaje de genes Bos taurus en el trópico mexicano. Téc Pecu Méx 2009; 47(4): 435-448.

15. Bourdon RM. Understanding animal breeding. 2nd ed. USA, NJ , Prentice-Hall, Inc. Upper Saddle River; 2000. 


\section{EFECTO DE LA PROPORCIÓN DE GENES SOBRE CARACTERÍSTICAS DE PESO AL DESTETE}

16. SAS. Institute, Inc. SAS/STAT Users Guide, version 9.2 ed. Cary, North Carolina, USA: SAS Institute Inc., 1997.

17. Neter J, Wasserman W, Kunter MH. Applied linear regression models. 2nd ed. USA, Irwin, Inc., 1989.
18. Mrode RA, Thompson R. Linear models for the prediction of animal breeding values. 2nd ed. USA, CABI Publishing, 2005.

19. The MathWorks, Inc., User MATLAB 7, Getting started guide, USA, The MathWorks, Inc. 2009. 
